\title{
Issues Behind Choosing Alternative Measures to Cancer Care and Cure
}

\author{
Abdul Kader Mohiuddin* \\ Treasurer, Dr. M. Nasirullah Memorial Trust, Tejgaon, Dhaka, Bangladesh \\ *Corresponding Author: Abdul Kader Mohiuddin, Treasurer, Dr. M. Nasirullah Memorial Trust, Tejgaon, Dhaka, Bangladesh.
}

Received: September 16, 2019; Published: September 23, 2019

DOI: 10.31080/ASMS.2019.03.0413

Regardless of extraordinary development, regular therapy misses the mark in cancer the board. There are two noteworthy obstacles in anticancer drug development: dose-limiting poisonous symptoms that diminish either drug viability or the QoL of patients and convoluted drug development forms that are exorbitant and tedious. Cancer patients are progressively searching out alternative medicine and may be hesitant to uncover its utilization to their oncology treatment doctors. Be that as it may, there is constrained accessible data on examples of use and viability of alternative medicine for patients with cancer. The cost of delivering cancer treatment is estimated to rise globally with a projected total spending of $\$ 458$ billion by 2030 [1]. However, the financial burden stems from employment loss, cost of care even when patients don't require chemotherapy, out of pocket costs' opportunity costs of informal care time and can continue long after the death of the patient. As adjuvant therapy, numerous conventional medicines demonstrated adequacy against mind, head and neck, skin, bosom, liver, pancreas, kidney, bladder, prostate, colon and blood cancers. Chemotherapy and radiotherapy are still generally customary methodologies for treatment of patients harboring propelled cancer. Customary chemotherapy likewise connected with neuropathic pain, fatigue and sleep aggravation, anxiety and depression, mouth sores, nausea and vomiting, early satiety, alopecia, bone and muscle squandering. Worldwide, some $60 \%-80 \%$ people depend on alternative medicines, which is also true for nearly $40 \%$ to $70 \%$ European, 50\% Italian, 40\% Korean, 30\% British and up to $87 \%$ of Australian cancer patients [2]. Use of unapproved/unlabeled/ wrong herbal treatment is not uncommon and also drug interactions reported phyto-therapeutics in oncology. Futile medicine use reported in one-fifth of terminally ill cancer patients (statins and antidementia drugs in nearly $100 \%$ cases, antihypertensives and bisphosphonates in nearly $30 \%$ cases) [3,4]. So, Proper and up-to-date knowledge is necessary in using alternative treatment options as patients who received alternative medicines had a 2.5 greater risk of dying compared to those who received conventional cancer treatment [5]. The objective of cancer palliative consideration is to forestall or treat, the manifestations and reactions of the cancer type and its treatment, providing care to any related physical, enthusiastic, social, and otherworldly angles. Pain influences more than $70 \%$ cancer patients, distressing or unbearable in more than one-third of patients and incessant pain is related with essential cancer itself or metastases or its treatment (interminable post-cancer treatment pain) [6,7]. In spite of the fact that, WHO depicted narcotics as fundamental medicines for pain control however appropriation demonstrates significant disparity, an under $10 \%$ of the total populace expending over $90 \%$ of the world's supply (80\% consumed by US alone) [8,9]. Additionally, some 85\% of essential consideration doctors apparent their preparation in pain the board to be insufficient in a Pan-European overview. Along with these, dread of reliance, remedy redirection, administrative examination, withdrawal side effects, narcotic related antagonistic occasions and death limit its utilization. There is an absence of superb proof with respect to the pain-relieving viability of NSAIDs in cancer; logical inconsistency and conflicting discoveries additionally revealed, despite the fact that pushed as a valuable aide for the board of cancer pain. Likewise, long-term utilization of NSAIDs is regularly associated with cardiovascular, gastrointestinal, renal, and other side effects [10]. Some different investigations likewise uncover relationship of NSAIDs with certain cancer types. A few investigations bolster utilization of cannabis/weed in cancer pain the executives. Its social agreeableness is step by step expanding far and wide, yet numerous examinations contradict its utilization or 
if nothing else demand further examination of advantage chance proportion. Some alternative treatments, similar to needle therapy, physical therapy, aromatherapy, CBT are generally suggested along with mind-body mediations like yoga, jujitsu, reflection and mindfulness, that keep individuals fit and vigorous as they experience treatment. $33 \%$ cancer patients utilize alternative medicine in US-are not all around controlled and may cooperate with regular medications like chemotherapy and radiation [11]. Mind-body mediations were each utilized by under $10 \%$ of patients, can keep individuals fit and vivacious as they experience treatment, decrease the symptoms of conventional treatments and improve patients' sleep, stress and emotional wellness. Numerous clinics even have alternative medicine centers that offer these projects.

\section{Bibliography}

1. Ghose S., et al. "Ethics of cancer care: beyond biology and medicine”. E Cancer Medical Science 13 (2019): 911.

2. Buckner CA., et al. "Complementary and alternative medicine use in patients before and after a cancer diagnosis". Current Oncology 25.4 (2018): e275-e281.

3. Oliveira L., et al. "Deprescription in Advanced Cancer Patients Referred to Palliative Care". Journal of Pain and Palliative Care Pharmacotherapy 30.3 (2016): 201-205.

4. Gonçalves F. "Deprescription in Advanced Cancer Patients". Pharmacy (Basel) 6.3 (2018): E88.

5. Johnson SB., et al. "Use of Alternative Medicine for Cancer and Its Impact on Survival". Journal of the National Cancer Institute 110.1 (2018).

6. Fallon M., et al. "ESMO Guidelines Committee. Management of cancer pain in adult patients: ESMO Clinical Practice Guidelines". Annals of Oncology 29.4 (2018): iv166-iv191.

7. Singh $\mathrm{P}$ and Chaturvedi A. "Complementary and alternative medicine in cancer pain management: a systematic review". Indian Journal of Palliative Care 21.1 (2015): 105-115.

8. Bhadelia A., et al. "Solving the Global Crisis in Access to Pain Relief: Lessons From Country Actions". American Journal of Public Health 29 (2018): e1-e3.

9. Rose ME. "Are Prescription Opioids Driving the Opioid Crisis? Assumptions vs Facts". Pain Medicine 19.4 (2018): 793-807.
10. Wong RSY. "Role of Nonsteroidal Anti-Inflammatory Drugs (NSAIDs) in Cancer Prevention and Cancer Promotion". Advances in Pharmacological Sciences (2019): 3418975.

11. Sanford NN., et al. "Prevalence and Nondisclosure of Complementary and Alternative Medicine Use in Patients with Cancer and Cancer Survivors in the United States". JAMA Oncology 5.5 (2019): 735-737.

\section{Volume 3 Issue 10 October 2019 \\ (c) All rights are reserved by Abdul Kader Mohiuddin.}

